\title{
A Study on Zigbee Based Human Body Monitoring System
}

\author{
Pranali Gaikwad ${ }^{1}$, Chetan Mane ${ }^{2}$, Madhuri Rodge ${ }^{3}$ \\ 1,2,3 Department of Electronics \& Telecommunication Engineering, \\ Shivajirao S. Jondhle College of Engineering \& Technology Asangaon, Maharashtra, India.
}

Abstract - This paper introduce us a real time body monitoring system. In now a day's security and health care becomes an hard issue. To take off and to monitor a patient continuously is very hard and huge task for doctors. This system plays an important role in monitor patients physical and mental health conditions. ZigBee can measure physical parameters like temperature, heart rate, blood pressure, patient's movement with the help of some devices either inserted in body or externally attached to human body. It helps to get correct or accurate readings from human body and passes it to ZigBee. Next step is to process the data. The data will be sent to hospital or doctor if in case of any emergency by ZigBee wireless module. The hospital and monitoring system receive all the information about patients and save this information into database from which they can judge the condition of patients by studying the monitoring records and patient's health. This data can be deployed in graphical form or in numerical form in dataset. According to the information collected from monitoring system the patient doctors having advantage to understand the patient condition more gracefully and also start the treatment according to continues information gathers. .

Keywords- ZigBee, Blood pressure, Heart rate, Motion sensor, Database, Health care.

\section{INTRODUCTION}

Wireless sensor network plays an important role in our day to day life. In our day to day life we used various types of wireless devices like smartwatch, wrist bands, earphones, etc. The main key point of wireless sensors is it can locate radio frequencies and adjust there power requirements all by itself also it can available in lower cost because of that it can result in further future developments in smart sensing devices. Wireless sensor networks mostly makes sensors on ZigBee technology. Which provides a small private wireless network. ZigBee can transmit the data with rate of $250 \mathrm{kbps}$.

The main advantages if ZigBee are-

1. It can run on low power.

2. Its cost is low as compared to other wireless devices.

3. It can provide free frequency bands etc.

The physical parameters like heart rate, blood pressure, temperature, etc. needs to monitor in real-time but if any goes to the doctor or hospital to check his condition, big instruments present over there which is traditional way to monitor your condition. The instruments are big in size and also, they are wired then its not possible to monitor a patient in real-time and also it will be very costly for a patient. All this facility are continuously available for only that patient which is in critical condition or in ICU and it will monitored by nurses. For normal patient also nurse is available for monitoring you, but she will only monitor you 2 to 3 times in a day. Because of that serious patients can't get help in time. With the help of ZigBee technology, it is possible to monitor a patient in real-time. With wireless sensor and a Zigbee module carrying with patient we can acquired real-time data. The monitored data can store on cloud storage. with the help of this monitored data, doctor can start his/her treatment immediately in any emergency situation. The main motive of this system is to find is patient is in worse condition or not and notify the doctor to help patients.

\section{RELATED WORKS}

The continuous health monitoring systems are essential for old-age people or critical conditions patients. But currently it is in progress to make system more compact and easier to carry with us[1].

Medical field have a wide scope of IOT in future. Patient interactions, patient monitoring cab be done by IOT. It will save times and cost of both patient and doctor[1][2].

On few cases if patient seen in worse condition then ZigBee/ system will inform the staff or doctor immediately. So, they can take immediate action on situation[4]

The data collected from Zigbee or sensors can be stored on both offline and online storage. The wearable devices can give information in both conditions, inside the hospital and at outside of hospital, also, this can help doctors to see real-time condition of patient.

It can also monitor the neural activities of patient with some additional sensors and Zigbee. 


\section{DETAILED DESCRIPTION}

The detailed description of system can be seen from two phases -

1. Block diagram

2. Algorithm

1. Block diagram -

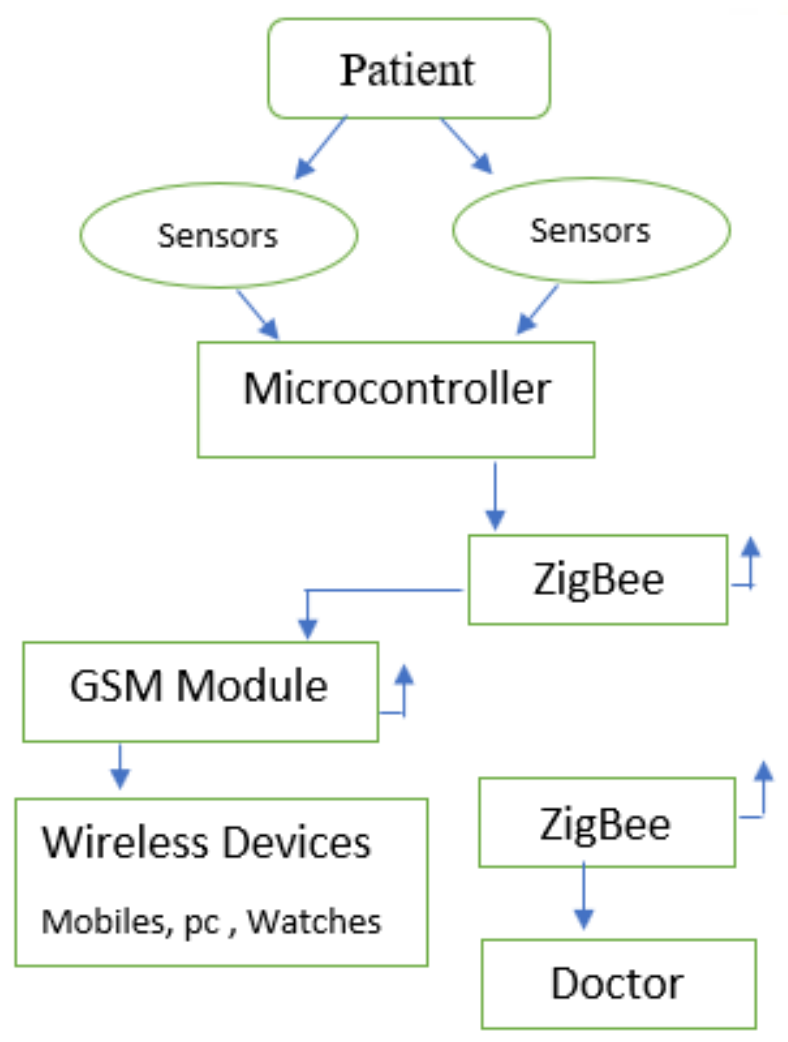

Description -

The sensors are placed inside patients body or attached on patients body externally. The microcontroller is can be used to make it easier to use. By using microcontroller, it can send the data directly to wireless devices with the help of GSM module or can be send to hospital staff or doctors pc through Zigbee.

Zigbee is the compact controller which consists pf transmitter and a receiver for communicating data/transmitting the data from microcontroller to doctor.

GSM module is used to send the urgent notification message over wireless devices in case of any emergency.

2. Algorithm -

- Attach sensors to patients body for monitoring purpose.

- Collect real-time data.

- Compare the data with standard values.
- The collected information then stored on online and offline both database.

- Then sends it to doctors PC or to nurses in hospitals.

- An alarm or notification will be send on doctors phone or in hospital in case of any emergency.

\section{DEVICE AND FUNCTION}

In monitoring of patients, different monitoring devices and sensors are used. Every sensor is connected to a microcontroller and a Zigbee.

An authorised person can get details of patients by login or any other security pass. These devices or sensors provides real-time records of the patient without any disturbance to the patient just because of it. These devices are very easy and comfortable to wear. These devices have very low in cost as compared to other devices. In some medical cases we needs accurate values/data records of patients in real-time. In that cases multiple numbers of sensors can use. All this data is then collected and transferred to controller.

Sometimes devices can provide different values from each other so, in such case doctor will decide which values are correct and which are not. This devices work independently but at last they all connected to a microcontroller through a Zigbee.

\section{- Zigbee -}

Zigbee is mostly used to create wireless personal area network for communication purpose. It is mainly used in small scale projects because of its low power consumption and low cost. Zigbee is mainly known for its security and data transfer speed parameters. But after encryption of data its security parameters will become low. Zigbee is also act as a transmitter and receiver at same time.

Zigbee is compact in size therefore it can be used as coordinator, router and in other end devices. And also batteries are inbuilt in Zigbee. Zigbee is also known for its level communication protocol.

After receiving the data, Zigbee encrypts this data for further transmission and also supports star, mesh and tree topologies. It can also receive multiple data from multiple devices without any loss in data. It can be use as internet support module and Wi-Fi connector.

- Sensors -

Sensors are used to take the accurate data of patient. In our system we can use $20-22$ sensors on single Zigbee.

\section{Motion sensor}

Motion sensors are the sensor which related to body movements. Motion sensors are attached to patient's hands or 
foot i.e. in foot ware and band in hand. and when this sensor activates means comes in motion, then it transmits the record to Zigbee. With the help of motion sensors, we can continuously monitor patient's movement. It includes distance travelled, speed of traveling, route of travelling.

\section{Heart rate sensors}

This sensor is placed near the chest or attached to any nerve where heart beats can be measured accurately. Heart rate sensors are very compact and easy to use.

\section{Blood pressure sensors}

This sensor is placed on arm, finger or attached to any nerve near arm where pulse can be measure accurately.

We can run at least 10-12 sensors continuously at a time on a single Zigbee sensor. And a single Zigbee sensor can transmit or receive any signal from 200M of distance.

\section{DATA MANAGEMENT}

\section{- Data storage -}

Dataset is created through a survey among doctors, from which a standard chart will create for further patient monitoring and all this information is stored on Zigbee cloud storage. All the data collected from doctors will be further encrypted for security purpose. In worst condition/situation of patient emergency tips will be sent to patient first. The report or information monitored will be compare from database which is on cloud then compared data will be sent on doctors pc. On Zigbee you can even create Login ID and passwords for security purpose from which it will only given to authorised persons, patients relatives, and patient itself. And for unauthorised person have to submit on request to patient to add login id and password to Zigbee system. Patient can daily monitor his physical condition and other things like heart rate, blood pressure, temperature etc.

\section{- Data sharing -}

The data sharing section is used in between patient and doctors only but some authorised person can also access the data about patients. Doctors can also hide some information from patient if any worse condition or in private concepts. Also it is applicable for patients relatives. This privacy can be modified by patient and doctor only.

\section{- $\quad$ Processing phase -}

In this phase, data analysis is done. Which is monitored from patients body and database will store every activity of patients. So this process compare real-time information with previous scored information. If any changes are observed, then the information will compare with doctors standard information which is stored on cloud storage of Zigbee. If ay critical condition observed, then an notification will be sent on doctors mobile using GSM module and to doctors PC and in hospital using Zigbee.

\section{FUTURE SCOPE}

In future it may possible to add advanced devices in Zigbee which could monitor patients neural activities.

There is also having scope for sensors can work without charging or without batteries. It can be possible to provide emergency medical help with the help of Zigbee only. It may also possible to kept emergency medicines with in Zigbee, so that patient can get medicine immediately.

\section{CONCLUSION}

This system can be implemented with some help of doctors and some hospitals to reduce the work load of doctors. This system can also helps doctors to be informed about patients before any emergency. The data which is deleted by doctors or patients are still available on cloud storage.

\section{REFERENCES}

[1]. A. Yassine, S. Singh and A. Alamri, Mining Human Activity Patterns From Smart Home Big Data for Health Care Applications, 2017 IEEE Access, (Vol. 5, pp. 13131-13141), IEEE.

[2]. Riazul, S. M.,Islam, Daehan Kwak, Md. Humaun Kabir, Mahmud Hossain, and Kyung-Sup Kwak.2015. The Internet of Things for Health Care: A Comprehensive Survey. 2015 IEEE (Vol.3.pp678-708), IEEE.

[3]. Samit, Majumder, Deen, M.,Jamal and Tapas,Mondal., 2017. Wearable Sensor for Remote Health Monitoring 17(1),pp.130- 139

[4]. Benny P.L. Lo, Surapa Thiemjarus, Rachel King and GuangZhong Yang. 2017 Body Sensor Network - A Wireless Sensor Platform for Pervasive Healthcare Monitoring 11(7),pp.378385.

[and Wei Che, . 2016. A Review of Wearable Sensor Systems for Monitoring Body Movements of Neonates 2016, pp. 21342151.

[5]. Shivani Bhattacharjee, Bhargav Gokalgandhi and Ameya Singh.,2014. Industrial Automation and Sensing using ZigBee volume 105,pp.87-95

[6]. Saed Tarapiah, Shadi Atalla and Salah Haj Ismail, 2016. Smart Real-Time Healthcare Monitoring and Tracking System using GSM/GPS Technologies, Computer Applications 16(12), pp 882-890.

[7]. Tatsuhiro Fujimoto, Hiroshi Nakajima, Naoki Tsuchiya, Hideya Marukawa, Kei Kuramoto, Syoji Kobashi, Yutaka Hata,2013. "Wearable Human Activity Recognition by Electrocardiograph and Accelerometer", Multiple-Valued Logic (ISMVL) 2013 IEEE 43rd International Symposium on, pp.12- 17, IEEE

[8]. Y. Hata, H. Yamaguchi, S. Kobashi, K. Taniguchi and H. Nakajima, A human health monitoring system of systems in bed, 2008 IEEE International Conference on System of Systems Engineering, Singapore, 2008, pp. 1-6,IEEE.

[9]. V. R. Singh, Advanced U-health care systems, 2017 6th International Conference on Informatics, Electronics and Vision \& 2017 7th International Symposium in Computational Medical and Health Technology (ICIEVISCMHT), Himeji, 2017, pp. 1- 1, IEEE. 
International Journal on Recent and Innovation Trends in Computing and Communication

ISSN: 2321-8169 Volume: 8 Issue: 5

DOI: https://doi.org/10.17762/ijritcc.v8i5.5396

Article Received: 07 April 2020 Revised: 26 April 2020 Accepted: 18 May 2020 Publication: 31 May 2020

[10].B. M. Huhman et al., Automated health monitoring of a pulsed power system, 2014 IEEE 41st International Conference on Plasma Sciences (ICOPS) held with 2014 IEEE International

Conference on High-Power Particle Beams (BEAMS),

Washington, DC, 2014, pp. 1-1, IEEE.

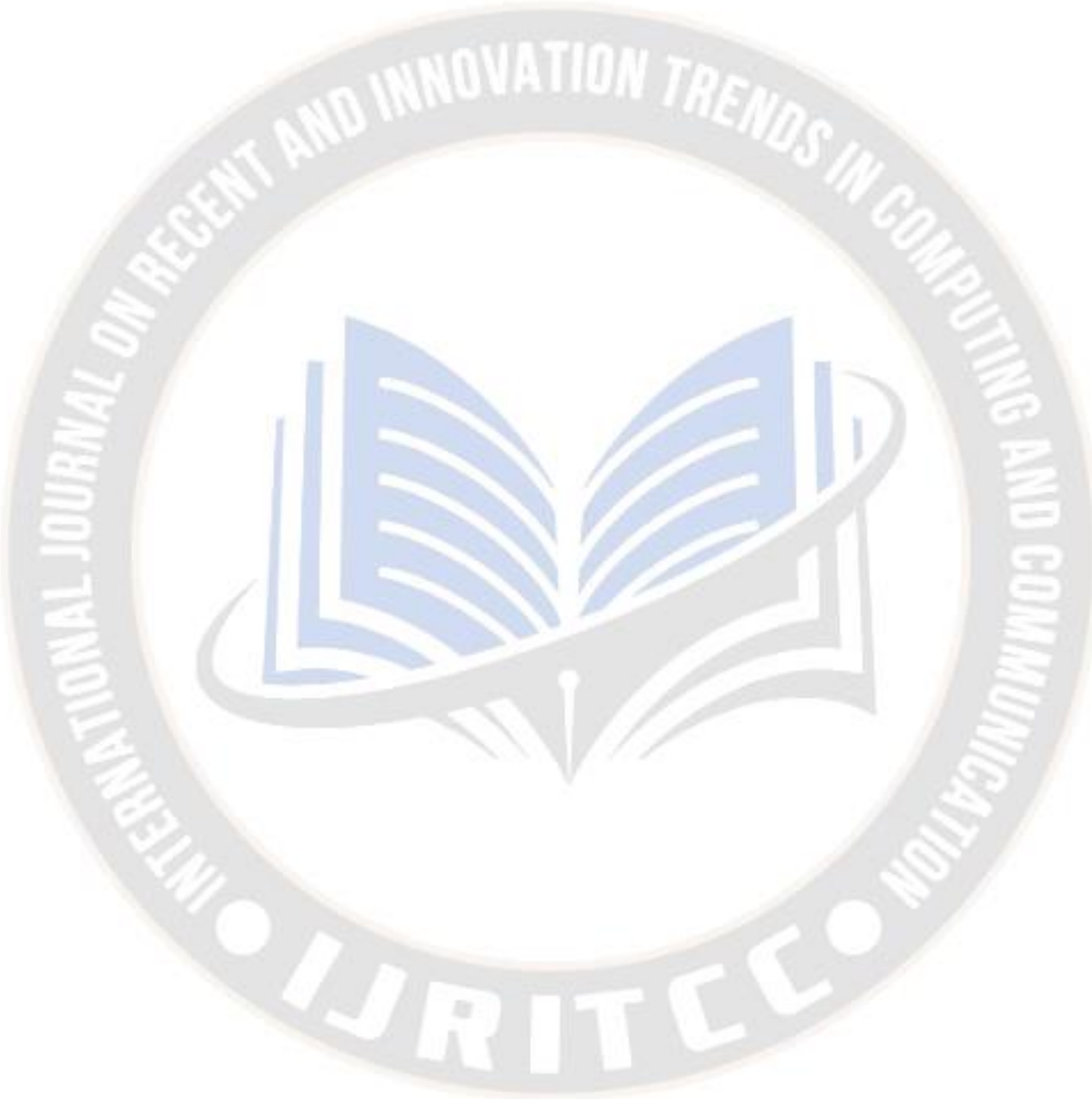

